\title{
O PRÊMIO NOBEL DE FÍSICA DE $2009^{+*}$
}

\author{
José Maria Filardo Bassalo \\ Fundação Minerva \\ Belém - PA
}

\section{Resumo}

Neste artigo, trataremos do Prêmio Nobel de Física de 2009, concedido aos fisicos norte-americanos: Charles Kuen Kao (nascido na China), por haver descoberto o processo de transmissão de luz em fibra óptica; e Willard Sterling Boyle (nascido no Canadá) e George Elwood Smith, pela invenção do primeiro sensor de imagem digital, um circuito semicondutor de imagem, conhecido como sensor $C D D$.

Palavras-chave: Prêmio Nobel de Física de 2009; Kao; Boyle e Smith; Transmissão de luz em fibra óptica; circuito semicondutor de imagens - o sensor CCD.

\begin{abstract}
In this article we will talk about the Nobel Prize in Physics 2009, granted to the north american physicists: Charles Kuen Kao (born in China), for his discovery of the light transmission process in optical fibers; and Willard Sterling Boyle (born in Canada) and George Elwood Smith, for the invention of the first digital image sensor, a semiconductor image circuit - the CCD sensor.
\end{abstract}

Keywords: 2009' Physics Nobel Prize; Kao; Boyle e Smith; light transmission in optical fibers; and semiconductor image circuit the CCD sensor.

\footnotetext{
+ The 2009 Physics Nobel Prize

* Recebido: fevereiro de 2010. Aceito: março de 2010.
} 
O Prêmio Nobel de Física (PNF) de 2009 foi compartilhado da seguinte maneira: metade ao físico norte-americano Charles Kuen Kao (n.1933) (nascido na China), pela sua descoberta de como transmitir luz por intermédio das fibras ópticas; e a segunda metade dividido entre os físicos, também norte-americanos, Willard Sterling Boyle (n.1924) (nascido no Canadá) e George Elwood Smith (n.1930), pela invenção do primeiro sensor de imagem digital, um circuito semicondutor de imagem, conhecido como sensor CDD (Charge-Coupled Device) (Dispositivo de Carga-Emparelhada). Vejamos como aconteceram essas descobertas.

Muito embora a passagem (certamente guiada por reflexão total) da luz em pedaços de vidros coloridos tivesse sido usada como decoração no Egito e na Mesopotâmia, cerca de milhares de anos antes de Cristo, a ideia de guiar a luz em um meio denso por intermédio da reflexão total, deve-se aos físicos, o suíço JeanJefe (Daniel) Colladon (1802-1893) e o francês Jacques Babinet (1794-1872) que, em 1842, e, independentemente, discutiram a possibilidade de guiar a luz em um jato de água ou mesmo em um tubo de vidro recurvado. Mais tarde, em 1870, o físico inglês John Tyndall (1820-1893) realizou uma experiência na qual guiava a luz em um jato de água que fluía entre dois recipientes. É oportuno registrar que a reflexão total da luz acontece quando ela passa de um meio mais denso para um meio menos denso, com um ângulo de incidência maior que o chamado ângulo limite: esse é o ângulo de incidência que corresponde a um ângulo de refração igual a $90^{\circ}$.

No começo do Século 20, a ideia de transmitir luz em um meio denso voltou a ser objeto de pesquisa. Com efeito, em $1910^{1}$, o físico e químico holandês Petrus Joseph Wilhelm Debye (1884-1966; PNQ, 1936) e o físico grego Demetrius Hondros (1882-1962) publicaram um trabalho teórico sobre a possibilidade de transmissão de luz em um guia de onda dielétrico. Mais tarde, em $1920^{2}$, O. Schriever publicou os primeiros trabalhos experimentais sobre a propagação de ondas eletromagnéticas em fios dielétricos, nos quais procurou testar os cálculos teóricos de Debye-Hondros. Em 1926, o engenheiro escocês John Logie Baird (1888-1946) patenteou a ideia de transmitir imagens através de um cabo de vidro flexível. Note que, também na década de 1920, um feixe de filamentos maciços de vidro era usado para investigar o aparelho digestivo humano ou como periscópios

\footnotetext{
${ }^{1}$ Annales de Physique Leipzig, v. 32, p. 465, 1910.

2 Annales de Physique Leipzig, v. 63, p. 645, 1920.
} 
flexíveis. Contudo, essas fibras de vidro eram muito fracas e não transmitiam muita luz e, às vezes, deixavam escapá-la pelo desgaste causado pelo atrito entre elas.

Estimulado pela experiência de Tyndall e considerando as deficiências da fibra de vidro apontadas acima, em 1952, o físico indiano Narinder Singh Kapany (n.1927) iniciou seus trabalhos que o levaram à invenção da fibra óptica: uma fibra de vidro revestida por um material cujo índice de refração é um pouco menor do que o do vidro. Por sua vez, em $1954^{3}$, o físico holandês Abraham Cornelis Sebastien van Heel (1899-1966) descreveu uma técnica para transmitir imagens ópticas sem aberração (perdas), empregando um guia dielétrico revestido com uma camada de material de baixo índice de refração. Seu objetivo estava relacionado apenas com o alinhamento de imagens. Aliás, nessa mesma Nature 173 e na mesma página 39, Kapany e o físico e matemático inglês Harold H. Hopkins (n.1918) (o inventor da lente zoom, em 1948) publicaram um trabalho no qual descreveram suas experiências com a transmissão de luz por intermédio de um dispositivo formado de um feixe de fibras ópticas; novas experiências com esse dispositivo foram descritas por Kapany e Hopkins, em $1955^{4}$. Nessas pesquisas, eles usaram esse dispositivo para aplicações citoscópicas, como por exemplo, examinar o trato (aparelho) digestivo humano. (Registre que Hopkins já pensara nesse problema em decorrência dos trabalhos que realizara, em 1943 e 1945, sobre a resolução de microscópios quando iluminado com luz polarizada.) Em 1957, Kapany publicou artigos no Journal of the Optical Society of America ${ }^{5}$, sobre a tecnologia de fibras ópticas que havia desenvolvido: isoladamente (p. 413); com J. A. Eyer e R. E. Keim (p. 423); com Hopkins (p. 594); e com J. N. Pike (p. 1109). Em $1961^{6}$, E. Snitzer apresentou uma proposta de como realizar uma ação de laser em uma fibra óptica. Essa proposta foi concretizada, em 1964 ${ }^{7}$, quando Snitzer e C. J. Koester conseguiram uma ação laser em uma fibra de vidro dopada com neodímio (Nd). É interessante notar que, na primeira metade da

\footnotetext{
${ }^{3}$ Nature, v. 173, p. 39, 1954.

${ }^{4}$ Óptica Acta, v. 4, p. 164, 1955.

5 Journal of the Optical Society of America, v. 47, 1957.

${ }^{6}$ Journal of Applied Physics, v. 32, p. 36, 1961.

${ }^{7}$ Applied Optics, v. 3, p. 1182, 1964.
} 
década de 1960, as fibras ópticas utilizadas apresentavam uma alta atenuação expressa em $\mathrm{db} / \mathrm{m}[\mathrm{db}=\mathrm{decibel}=\mathrm{b} / 10$; o bel (b) é uma unidade adimensional que exprime o quociente entre duas potências (elétrica ou acústica), sendo uma de referência; o número de bels é dado pelo logaritmo decimal daquele quociente]. Por exemplo, apenas cerca de um por cento (1\%) de luz era transmitida em cada 20 metros de fibra. Em vista disso, outras tecnologias de transmissão óptica foram então sendo desenvolvidas.

Agora, vejamos o trabalho de Kao que o levou a ganhar a metade do PNF2009. Kao se graduou em Engenharia Elétrica na Universidade de Londres, em 1957, e nessa mesma Universidade defendeu sua Tese de Doutorado (QuasiOptical Waveguides), em 1965, sob a orientação do físico australiano Antoni Emil Karbowiak (n.1923), que dirigia um grupo de pesquisas em comunicação óptica no Standard Telecomunications Laboratories Ltd. (STL), em Harlow, na Inglaterra, no qual Kao trabalhava desde 1960. Observe que o STL era uma subsidiária da indústria norte-americana International Telephone and Telegraph Corporation (ITT). Quando Karbowiak decidiu voltar para a Austrália, sugeriu a Kao que iniciasse um pequeno grupo de pesquisas para investigar o mecanismo de dissipação de materiais dielétricos usados em fibras ópticas. Kao passou então a trabalhar com o físico e engenheiro inglês George Alfred Hockham (n.1938); este, junto com Karbowiak, no STL, desde 1961, estudava as perdas em fibras dielétricas [com diâmetro da ordem $1 \mu \mathrm{m}\left(=10^{-6} \mathrm{~m}\right)$ ], em decorrência das descontinuidades das mesmas e de impurezas. Para contornar essa perda, Hockham revestiu as fibras de dielétricos com um material transparente, ou seja, com dado índice de refração. Então, Kao e Hockham começaram a pesquisar não só a Física dessas guias, mas, principalmente, as propriedades dos materiais usados em sua fabricação. Kao, por sua vez, resolveu investigar as perdas em vidro para ver se as mesmas podiam ser diminuídas. Assim, em junho de $1966^{8}$, eles publicaram o artigo intitulado Dielectric-Fibre Surface Waveguides for Optical Frequencies, no qual propuseram a comunicação óptica através de fibras de vidro revestidas. É oportuno destacar que, em setembro de $1966^{9}$, A. Werts fez uma proposta análoga para fibras de vidro, no qual citou o artigo de Kao e Hockham, quando discutiu a perda óptica nessas fibras.

\footnotetext{
${ }^{8}$ Proceedings of the Institute of Electrical and Electronic Engineers, v. 113, p. 1151, 1966.

${ }^{9}$ L'Onde Electrique, v. 46, p. 967, 1966.
} 
No artigo que Kao escreveu com Hockham referido acima, Kao afirmou que:

uma fibra de material de vidro construida em uma estrutura revestida [...] representa uma possivel guia de onda óptica prática com importante potencial como uma nova forma de comunicação [...] Comparada com os cabos co-axiais usados em sistemas de radiocomunicação, esta nova forma de guia de onda tem uma grande capacidade de informação e possíveis vantagens de custo material.

Tendo como base essa premonição, Kao continuou suas pesquisas no STL, agora com novos parceiros, T. W. Davies, M. W. Jones e C. R. Wright, tentando diminuir as impurezas do vidro [principalmente íons de ferro $(\mathrm{Fe})]$ e fazendo cuidadosas medidas da atenuação da luz, nesses vidros purificados, e em outros materiais, usando diferentes comprimentos de onda. Como resultado desse trabalho, eles publicaram artigos em $1968^{10}$ e em $1969^{11}$.

Nessas pesquisas, Kao descobriu que a sílica [dióxido de silício $\left(\mathrm{SiO}_{2}\right)$; material transparente, parecido com o vidro] fundida tinha uma pureza compatível com a comunicação óptica que ele previra. Contudo, como esse material apresentava uma alta temperatura de fusão e, portanto, de difícil fabricação e manipulação, os laboratórios de Fibras Ópticas mundiais preferiam usar o vidro, porém sem muito sucesso, já que a perda continuava sendo grande. Porém, em $1970^{12}$, os físicos norte-americanos F. P. Kapron, Donald D. Keck e Robert D. Maurer (n.1924), pesquisadores do Corning Glass Works, nos Estados Unidos (sob a liderança de Maurer), anunciaram que haviam desenvolvido o método químico CVD (Chemical Vapor Deposition) e com ele construíram fibras ópticas de sílica e revestiram-na com sílica fundida. Contudo, para que o caroço ("core") da fibra e seu revestimento tivessem índices de refração próximos, eles doparam o caroço com titânio (Ti). Com essa fibra, eles conseguiram uma perda de $20 \mathrm{db} / \mathrm{km}$. Mais tarde, em $1973^{13}$, Keck, Maurer e P. C. Schultz anunciaram que haviam conseguido uma nova fibra óptica ainda com sílica, com o caroço, porém dopado com

\footnotetext{
${ }^{10}$ Journal of Scientific Instruments, v. 1, p. 1063, 1968.

${ }^{11}$ Journal of Scientific Instruments, v. 2, p. 331; 579, 1969.

${ }^{12}$ Applied Physics Letters, v. 17, p. 423, 1970.

${ }^{13}$ Applied Physics Letters, v. 22, p. 307, 1973.
} 
germânio (Ge), e uma perda de $4 \mathrm{db} / \mathrm{km}$, para luz de $\lambda=850 \mathrm{~nm}\left(1 \mathrm{~nm}=10^{-9}\right.$ $\mathrm{m})$.

Por outro lado, nas décadas de 1970 e 1980, nos Laboratórios Bell, os físicos norte-americanos John B. MacChesney (n.1929) e P. B. O'Connor modificaram o CVD, criando o MCVD (Modified Chemical Vapor Deposition), o que the permitiram inventar um gel de alta pureza para revestir o caroço de sílica usada na fibra óptica. Esse trabalho rendeu a eles as seguintes Patentes: US444705 (22/02/1974); US828617 (29/08/1977); US117522 (01/02/1980); US217027-A (12/08/1980); US559729 (09/12/1983) e US4217027-B (15/07/1986). É interessante registrar que, no final da Nobel Lecture de Kao, lida por sua mulher Gwen M. W. Kao, no dia 08 de dezembro de 2009, ela afirma que: "Charles Kao plantou a semente, Robert (Bob) Maurer regou e John MacChesney cresceu suas raízes".

Registre-se que, em 1988, foi instalado o primeiro cabo transatlântico de fibra óptica, que tinha a capacidade para 40.000 conversas telefônicas simultâneas. Hoje (fevereiro de 2010), com uma atenuação abaixo de $0,2 \mathrm{db} / \mathrm{km}$, para luz de $\lambda=1,55 \mu \mathrm{m}\left(1 \mu \mathrm{m}=10^{-6} \mathrm{~m}\right)$, as fibras ópticas, além de serem usadas em comunicação, possuem um grande número de aplicações: medicina, tecnologia laser e sensores. Como exemplo de aplicação das fibras ópticas na comunicação científica, é oportuno destacar que no Conseil Européen pour la Recherche Nucléaire (CERN), na fronteira entre França e Suíça, foi desenvolvido uma rede de fibras ópticas altamente avançada para permitir que os dados obtidos no LHC (Large Hadron Collider) pudessem ser transmitidos para o mundo inteiro. Note que, para transmitir dados por uma fibra óptica, é necessário um fotoemissor [que pode ser um diodo emissor de luz (Light Emissor Diode - LED, que produz luz incoerente) ou um diodo laser (que produz luz coerente), ambos inventados em 1962] para converter sinais elétricos em pulsos de luz que representam os valores digitais binários: 0 e $1^{14}$.

Tratemos, agora, das contribuições de Boyle e Smith que os levaram a ganhar a segunda metade do PNF2009, pela invenção do sensor CDD, importante para a invenção da câmara digital. Antes, no entanto, vejamos o desenvolvimento da fotografia para entendermos o significado dessas invenções. Em 1685, o óptico alemão Johann Zahn (16351-1707) publicou o livro intitulado Oculus Artificials

${ }^{14}$ R. G. W. Brown and E. R. Pike, In: Twentieth Century Physics III (Institute of Physics Publications and American Institute of Physics Press, 1995); The Royal Swedish Academy of Sciences, Scientific Background on the Nobel Prize in Physics 2009 (06 de outubro de 2009); Maurício Pamplona Pires, Ciência Hoje, dezembro de 2009; <pt.wikipedia.org/wiki/Fibra_Optica//Laser_Diodo> Acesso em: 05 jan. 2010. 
Teledioptricus Sive Telescopium ("Teledióptrico Óculos Artificial ou Telescópio"), no qual ilustrou, por intermédio de diagramas e esboços, vários dispositivos ópticos, como, por exemplo, a câmara escura (já conhecida pelas antigas civilizações como, por exemplo, a chinesa, a egípcia e a grega: um buraco feito em uma parede produzindo uma imagem invertida na parede oposta de uma sala escura), a lanterna mágica [inventada pelo cientista alemão, o jesuíta Athanasius Kircher (c.1602-1680), em 1646] e o retroprojetor [ou projetor de filmes (slides): um sistema óptico formado de lentes e um espelho côncavo, que concentra a luz - vinda de uma lâmpada colocada na frente do espelho - sobre uma transparência (slide) e a projeta em uma tela (screen) colocada a certa distância]. Mais tarde a câmara escura foi aperfeiçoada e tornou-se um instrumento portátil, com lentes e linguetes variáveis para tornar nítida, em um vidro fosco, a imagem de um determinado objeto. Contudo, não se sabia como fixar essa imagem. Em 1724, o químico e filósofo alemão Johann Heinrich Schulze (1867-1744) descobriu que certos "sais de prata", notadamente o cloreto de prata $(\mathrm{AgC} \ell)$ e o nitrato de prata $\left(\mathrm{AgNO}_{3}\right)$, escureciam na presença da luz solar. Ele próprio havia gravado palavras com a luz do Sol, que logo se apagavam; porém ninguém havia conseguido gravar a imagem, em definitivo, o que só ocorreu no Século $19^{15}$.

A ideia de gravar imagens foi retomada no final do Século 18. Com efeito, desde 1793, o litógrafo francês Joseph Nicéphore Niépce (1765-1833) e seu irmão Claude Niépce (oficial da Marinha Francesa), tentaram obter imagens gravadas quimicamente usando uma câmara escura com uma pequena lente convexa. Contudo, as imagens obtidas desapareciam rapidamente. Depois de alguns anos de pesquisa, Joseph conseguiu, em 1816, substituir o vidro fosco por papel com cloreto de prata $(\mathrm{AgC} \ell)$ e, com isso, fixou parcialmente as imagens, porém, não pôde passar seus negativos para positivos. Mais tarde, em 1822, Joseph conseguiu recobrir uma placa de estanho (Sn) com um tipo de asfalto chamado betume-da-judeia e que endurecia quando atingido pela luz. Por fim, em 1826, Joseph expôs uma dessas placas por cerca de oito (8) horas em uma câmara escura [fabricada pelo óptico francês Charles Chevalier (1804-1859)], com algumas adaptações. Essa câmara media $6 \times 8$ polegadas, e era constituída de duas caixas de madeira, sendo a parte traseira com um vidro fosco, deslizando dentro da parte da frente, que tinha o painel de lentes e que media $30 \mathrm{~cm}^{2}$ quando fechada. Essas duas partes eram ligadas por um fole tendo um diafragma em uma delas, funcionando

15 pt.wikipedia.org/wiki/Johann_Zahn // Johann_Heinrich_Sculze // Athanasius_Kircher. Observe que // significa outro site da wiki. 
como uma íris variável. Em 1829, Joseph substituiu as placas de metal revestidas de $\mathrm{Sn}$ e escureceu as sombras com vapor de iodo (I). Como Joseph era um litógrafo, deu a esse dispositivo o nome de heliógrafo, decorrente do vocábulo heliografia, que significa "gravura com luz solar", uma vez que a palavra helius quer dizer Sol, em grego ${ }^{16}$.

Com a morte de Joseph Niépce, em 1833, o prosseguimento de seus experimentos fotográficos foi conduzido por seu sócio, o pintor, cenógrafo e químico francês Louis Jacques Mande Daguerre (1787-1851), que havia descoberto, em 1831, a sensibilidade da prata iodada à luz. Mais tarde, em 1835, Daguerre descobriu, acidentalmente, que uma placa de prata sensibilizada com iodeto de prata (AgI) era revelada pela ação do mercúrio $(\mathrm{Hg})$, que estava no armário no qual ele displicentemente havia guardado aquela placa. Apesar de Daguerre haver padronizado esse processo, o mesmo apresentava grandes dificuldades: o tempo de exposição era longo (15 a 30 minutos), a imagem era invertida, e o contraste era muito baixo. Além disso, a imagem formada na chapa, depois de revelada, continuava sensível à luz solar e rapidamente era destruída. Esses problemas foram contornados por Daguerre, em 1837, ao descobrir que, mergulhando as chapas reveladas numa solução aquecida de sal de cozinha [cloreto de sódio $(\mathrm{NaC} \ell)$ ], que tinha um poder fixador, manteve inalterada a imagem obtida. Note que esse processo descoberto por Daguerre (mais tarde conhecido como daguerreótipo), foi por ele vendido ao Governo Francês em 1839. Em decorrência dessa venda, Daguerre passou a ter uma renda vitalícia anual de 6.000 francos e Isidore Niépce (1786-1868), filho de Joseph (que fora sócio de Daguerre, como vimos), recebia 4.000 francos também anuais ${ }^{17}$.

Esse processo de fixação de imagens - a fotografia - também foi desenvolvido na Inglaterra, ainda no Século XVIII. Com efeito, em 1835, o químico inglês William Henry Fox Talbot (1800-1877) inventou um papel fotossensível, que havia sido mergulhado alternadamente em soluções de nitrato de prata (Ag$\left.\mathrm{NO}_{3}\right)$ e cloreto de sódio $(\mathrm{NaC} \ell)$, criando o cloreto de prata $(\mathrm{AgC} \ell)$. Quando exposto à luz, esse cloreto criava uma imagem negativa, que podia ser exposta novamente e impressa em uma imagem positiva. Em 1840, Talbot melhorou seu processo, o calótipo (depois, talbótipo), e recebeu, em 1841, sua patente. É opor-

\footnotetext{
${ }^{16}$ pt.wikipedia.org/wiki/Joseph Nicephore Niepce; Helmut Gernsheim. In: Eureka! Uma História das Invenções (Editado por Edward Bono, Editorial Labor do Brasil S.A., 1975); Tom Philbin, As 100 Maiores Invenções da História (DIFEL, 2006).

${ }^{17}<$ pt.wikipedia.org/wiki/Louis_Jacques-Mande_Daguerre>.
} 
tuno registrar que, entre 1844 e 1846, Talbot publicou o livro The Pencil of Nature ("O Lápis da Natureza”), que foi o primeiro livro com fotografias. Segundo Philbin (op. cit.), a inovação do processo de Talbot foi o fato de que um mesmo negativo podia ser utilizado para imprimir múltiplas imagens positivas, diferentemente do daguerreótipo, que criava uma única cópia, impossível de ser duplicada. Contudo, o talbótipo apresentava um inconveniente: seus negativos apresentavam qualidade inferior da dos daguerreótipos, uma vez que os detalhes eram afetados pela fibra de papel utilizada no positivo.

As placas fotográficas (daguerreótipos e talbótipos) apresentavam um problema, pois eram feitas de vidro e precisavam ser preparadas pouco antes da exposição e reveladas imediatamente após; daí o nome original de fotografia com placa úmida. Esse problema foi contornado, por volta de 1870, com a invenção da chapa seca (dry plate), uma suspensão gelatinosa de brometo de prata $(\mathrm{AgBr})$ que poderia ser preparada antes da exposição e reveladas muito depois. Esse processo era cerca de 60 vezes mais rápido do que o talbótipo. Um dois pioneiros nessa nova técnica fotográfica foi o inventor norte-americano George Eastman (18541932), ao criar, em 1880, a Eastman Dry Plate and Film Company (transformada em Eastman Kodak Company, em 1892) e, em 1888, colocar no mercado a primeira câmera Kodak, com o seguinte slogan: "Aperte o botão e deixe que faremos o resto”. Essa câmara possuía um rolo de papel fotográfico [inventado pelo padre inglês Hannibal Goodwin (1892-1900), em 1887] para 100 exposições.

Outro grande avanço na técnica fotográfica ocorreu com a descoberta da fotografia colorida. Sua ideia inicial foi proposta pelo pianista e inventor francês Louis Arthur Ducos du Hauron (1837-1920), ao sugerir, em 1862, o chamado "método subtrativo", baseado no fato de que um pigmento colorido absorve todas as cores exceto a sua própria, que a reflete. Em 1868, ele obteve a patente francesa desse seu método (Fr83061), que usava as propriedades aditivas das cores fundamentais (vermelho, verde e azul) e as subtrativas das anticores fundamentais (cyan, magenta e amarelo). Essa sua invenção foi descrita no livro intitulado Les Couleurs en Photographie ("As Cores em Fotografias"), publicado em 1869. Note que a primeira fotografia colorida foi tirada por du Hauron, em 1877, de uma vista de Aven, no sudoeste da França, onde se encontra a Cathédrale Saint-Caprais. Uma nova ideia para o desenvolvimento da fotografia colorida aconteceu em 1890, quando o físico alemão Otto Wiener (1862-1927) desenvolveu uma técnica com a qual obteve ondas luminosas estacionárias, em que os nós e os antinós eram separados em intervalos de $\sim 2 \times 10^{-5} \mathrm{~cm}$. Usando essa técnica, o físico francês 
Gabriel Jonas Lippmann (1845-1921; PNF, 1908), em $1892^{18}$, apresentou sua invenção da fotografia colorida. Nesse processo inventivo (conhecido como processo interferométrico), a Ag contida em placas adredemente preparadas era separada em planos paralelos por ondas luminosas estacionárias. Quando esses planos eram vistos à luz do dia, os mesmos transmitiam ao olho somente aquelas cores que têm comprimento de onda que se iguala à distância entre esses planos, enquanto os outros comprimentos de onda eram eliminados por interferência luminosa [registre que esse fenômeno físico havia sido descoberto, em 1801, pelo físico e médico inglês Thomas Young (1773-1829)]. Em 1894 ${ }^{19}$, Lippmann publicou novos trabalhos sobre a sua invenção. [Gabriel Lippmann, Nobel Lecture (14 de dezembro de 1908)].

A técnica desenvolvida por du Hauron, em 1869, foi usada pelos músicos norte-americanos Leopold Godowsky, Jr. (1900-1983) (também químico) e Leopold Damrosch Mannes (1899-1964) para inventarem o filme Kodachrome, patenteado em abril de 1935 (U.S. Patent 1.997.493) (solicitada em janeiro de 1922). Ele consta, basicamente, de três emulsões luminosas sensitivas para a luz: camada de cima - azul, camada do meio - verde, e camada inferior - vermelho. Depois segue uma sequência de cores subtrativas: amarelo, magenta e cyan, para formar o negativo. Para maiores detalhes, ver a referência ${ }^{20}$.

Agora, vejamos os trabalhos nobelísticos de Boyle e Smith, realizados nos Laboratórios Bell, em New Jersey, Estados Unidos. Boyle defendeu sua Tese de Doutoramento em Física na McGill University (Canadá), em 1950, e foi para a Bell, em 1953; Smith doutorou-se também em Física na Chicago University, em 1959, e neste mesmo ano foi trabalhar na Bell. Na década de 1960, Boyle era o Diretor Executivo do Laboratório de Desenvolvimento de Dispositivos Semicondutores (hoje, Divisão de Ciências da Comunicação) e Smith era Chefe de um de seus Departamentos (hoje, o de Integração em Escala Muito Grande). Em 1967, F. Sangster havia patenteado um dispositivo que transportava cargas elétricas entre sucessivos capacitores por meio de transistores que transferiam o sinal armazenado ao capacitor vizinho uma vez em cada ciclo horário. Esse dispositivo foi cu-

\footnotetext{
${ }^{18}$ Comptes Rendus Hebdomadaires des Séances de l'Académie des Sciences, v. 115, p. 575,1892 .

Comptes Rendus Hebdomadaires dês Séances de l'Académie des Sciences, v. 118, p. 92, 1894; Journal de Physique, v. 3, p. 97, 1894.

${ }^{20}$ <pt.wikipedia.org/wiki/Leopold_Godowsky_Jr. // Leopold Mannes>
} 
nhado como BBD (bucket brigade device - dispositivo de uma brigada de baldes - em analogia a uma fila de pessoas transferindo baldes de água), por G. Krause, ainda em 1967, muito embora William J. Hannan já houvesse pensado nesse conceito de transferência de cargas, em 1965. Esse dispositivo de transferência de cargas foi descrito, em $1969^{21}$, por Sangster e K. Teer, que trabalhavam nos Philips Research Laboratories, na Inglaterra. É interessante destacar que, entre 1980 e 1988, Sangster obteve patentes desse seu invento em diversos países do mundo.

Contudo, a eficiência de transferir bolhas de cargas (charge bubbles) usando o BBD era limitada pelo comprimento da cadeia de transistores. Desse modo, segundo Smith descreveu em artigo publicado em $2009^{22}$, em 17 de outubro de 1969, ele foi ao gabinete de Boyle e, juntos, no quadro negro e por quase uma hora, esquematizaram a estrutura básica do sensor CCD que seria usado para transferir cargas elétricas. Basicamente, o CCD é um capacitor do tipo Si-MOS (silício-metal-oxide semiconductor) que registra imagens na forma eletrônica e, portanto, podem ser usadas em um filme fotográfico. Com efeito, quando a superfície do semicondutor [Si, dopado com boro (B) para transformá-lo em um portador do tipo p] recebe luz, há formação de bolhas de cargas em virtude do efeito foto-elétrico. [Lembrar que a banda proibida (entre a banda de condução e a banda de valência) do Si vale $1,14 \mathrm{eV}$; assim, fótons com energia acima desse valor poderá liberar elétrons, segundo a teoria proposta pelo físico germano-norteamericano Albert Einstein (1879-1955; PNF, 1921), em 1905]. O problema que então se apresentou a eles foi o de encontrar um modo engenhoso de transportar essas cargas de maneira ordenada.

Em vista do exposto acima, Boyle e Smith então idealizaram o seguinte: na estrutura superficial CCD foi colocada uma série de diminutos fios (eletrodos) formando uma matriz de pequenas células foto-sensitivas (hoje, elas são chamadas de pixels e têm dimensões $\sim 10 \mu \mathrm{m}$ ) dispostas em linhas e colunas. [Note que esses pixels contidos nos capacitores MOS correspondem aos grãos ("sais de prata") dos filmes fotográficos]. Portanto, quando a luz atinge esses pixels, são produzidos fótons-elétrons cujo número é proporcional à intensidade da luz utilizada. Desse modo, a distribuição de cargas nos pixels é uma representação análoga à de uma imagem. Assim, variando a voltagem (tensão elétrica) sobre essas células, as

\footnotetext{
${ }^{21}$ Institute of Electrical and Electronic Engineers (IEEE) - Journal of Solid-State Circuits, SC-4, p. 131, 1969.

${ }^{22}$ Nuclear Instruments and Methods in Physical Research A607, p. 1, 2009.
} 
"fotos-cargas" são retiradas em fila, uma coluna após a outra, e a imagem pode ser reconstruída. A invenção do sensor CCD foi apresentada, em 1970, em três artigos: um descrevendo a parte teórica: Boyle e Smith, Charge Coupled Semiconductor Devices ${ }^{23}$, e dois experimentais: Gilbert F. Amelio, M. F. Tompsett and Smith, Experimental Verification of the Charge Coupled Device Concept ${ }^{24}$; e Tompsett, Amelio and Smith, Charge Coupled 8-Bit Shift Register ${ }^{25}$.

Como o CCD transforma uma imagem em uma série de pulsos elétricos, logo se observou que ele poderia ter diversas aplicações tecnológicas, dentre elas, a câmara digital (CD), inventada em 1981. Mais tarde, em 1984, a Sony Corporation lançou no mercado a MAVICA (Magnetic Video Camera). A CD foi (e é) usada em investigações astrofísicas e astronômicas, como aconteceu com o The Hubble Space Telescopy, lançado em 24 de abril de 1990, pela National Aeronautics and Space Administration (NASA), equipado com diversas CD para diferentes propósitos. Por exemplo, a Wide Field Planetary Camera (WFPC) (Câmara Planetária de Largo Campo) usada para fotografar, em 2000, aglomerados de galáxias, como a Abell 2218, com a segunda versão dessa câmara (WFPC2). É, ainda, interessante destacar que a CD também foi (e é) utilizada em medicina, no estudo de tecidos e células, por intermédio de microscópios, endoscópios ${ }^{26}$ e scanners. Para mais detalhes sobre o CCD e suas diversas aplicações, ver referência ${ }^{27}$.

Concluindo este artigo, é interessante destacar que a invenção do circuito integrado (CI) pelo engenheiro eletrônico norte-americano Jack St. Clair Kilby (n.1923; PNF, 2000), em março de 1959, foi importante para o invento de diversos tipos de sensores de imagens, como o CMOS (complementary-metal-oxidesemiconductor), este em $1968^{28}$ e em $1969^{29}$, e o CCD (visto neste artigo), em 1970.

\footnotetext{
${ }^{23}$ Bell Systems Technical Journal, Briefs 49, p. 587, 1970.

${ }^{24}$ Bell Systems Technical Journal, v. 49, p. 593, 1970.

${ }^{25}$ Applied Physics Letters, v. 17, p. 111, 1970.

26

${ }^{26}<$ www.medscape.com $>$

${ }^{27}$ The Royal Swedish Academy of Sciences, op. cit.; Pires, op. cit.

${ }^{28}$ Peter J. W. Noble, Institute of Electrical and Electronic Engineers (IEEE) - Transactions on Electron Devices ED-15, p. 202, 1968.
} 
${ }^{29}$ P. K. Weimer, W. S. Pike, G. Sadasiv, F. V. Shallcross and L. Meray-Horvath, Institute of Electrical and Electronic Engineers (IEEE) - Spectrum, v. 6, p. 52; Savvas G. Chamberlain, Institute of Electrical and Electronic Engineers (IEEE) - Journal of Solid-State Circuits SC-4, p. 333, 1969. 